\title{
Enzymes in Organic Solvents: the Use of Lipases and $(R)$-Oxynitrilase for the Preparation of Products of Biological Interest*
}

\section{Vicente Gotor}

Departamento de Química Orgánica e Inorgánica. Facultad de Química. Universidad de Oviedo. 33006. Oviedo (España)

\begin{abstract}
The use of enzymes in organic solvents has acquired a special relevance in organic synthesis, and lipases are the enzymes most commonly used in transesterication reactions. In the past few years, we have shown the utility of enzymatic aminolysis and ammonolysis reactions for the preparation of amides and for the resolution of esters and amines. The enzymatic alkoxycarbonylation reaction is of great utility in chemoselective reactions of natural products. Lipases, enzymes much less exploited in organic synthesis, have an increasing interest, especially the use of $(R)$-oxynitrilases for the synthesis of optically active cyanohydrins.
\end{abstract}

Over the past few years our group has been working on the use of aminolysis and ammonolysis reactions for the synthesis of high value added products. We have studied the preparation of amides from $\alpha, \beta$-unsaturated esters, $\beta$-hydroxyesters or $\beta$-ketoesters with excellent results, thus achieving a very simple way of chemoselectively preparing different types of amides. If racemic amines are used, the corresponding amides are prepared in very high enantiomeric excesses. The enzymatic ammonolysis of diethyl 3-hydroxyglutarate allows the preparation of the corresponding amidoester in enantiopure form, a product we have used as starting material for the preparation of $(R)$-4-amino-3hydroxybutanoic acid, the precursor of carnitine. On the other hand, this methodology has allowed us to carry out resolutions of some heteroarylamines or different esters, offering an alternative to hydrolysis or enzymatic transesterification.

Recently we have accomplished a double resolution of esters and amines that allows the preparation of these substrates in enantiopure form in one step, since when the lipase of Candida antarctica (CAL) is used, the reaction takes place with high diastereoselectivity and enatioselectivity. Equally, when a prochiral ester and racemic amines are used the corresponding amidoester is prepared and the amines are resolved with enantiomeric exccesses grater than $95 \%$.

The best conditions for the resolution of trans-1,2-cyclohexanediamine involve using diesters and CAL as the biocatalyst. For example, when diethylmalonate is used both substrate and product are prepared in practically enantiopure form. From both of these we have developed a very efficient method for preparing different types of azamacrocycles. This process has great synthetic value, as it allows us 
to prepare different chiral macrocyles, compounds of great importance, as some of them have interesting properties concerning the recognition of chiral dianions.

As for the usefulness of enzymes in organic solvents in chemoselective transformations of natural products, our most outstanding results have been in the area of the nucleosides, especially with desoxyribonucleosides, and we are now investigating the possible applications of this methodology towards the synthesis of vitamin D. For this reason we have studied enzymatic acylation and alkoxycarbonylation reactions, particularly the latter, since they allow the regioselective preparation of carbonates which are suitable starting materials for the synthesis of other derivatives

In our studies of the enzymatic reactions of desoxyribonucleosides using esters or oxime carbonates we have achieved regioselective ayclation and alkoxycarbonylation at the 3' y 5' positions of the sugar. Thus, when the lipase of Pseudomonas cepacia (PSL) is used, acylation or alkoxycarbonylation at the 3' position is achieved, while, on the other hand, if CAL is used, the corresponding 5' isomers are prepared. The products obtained when the vinyloxycarbonyl group is introduced are starting materials for the preparation of nucleoside derivatives as yet not reported in the literature.

Presently we are studying different chemoenzymatic transformations in precursors of the A ring of $1 \alpha, 25$-dihydroxyvitamin $\mathrm{D}_{3}$, which is the hormonally active form of vitamin $\mathrm{D}_{3}$, as well as in the stereoisomers of the natural product, due to the importance of the stereochemistry of the chiral centers of this ring towards its biological responses. This allows us to prepare new derivatives of this important synthon through enzymatic alkoxycarbonylation reactions.

Another line of investigation in our group involves the use of oxynitrilases for the preparation of chiral cyanohydrins. $(R)$-oxynitrilase is a flavoprotein that catalyzes the addition of hydrogen cyanide onto the $s i$ face of benzaldehyde. The enzyme can be produced from almond flour and it can be used in different organic solvents, thus allowing it to react with a wide variety of aliphatic and aromatic aldehydes, and even ketones.

Recently we have investigated the cyanation-transcyanation reactions of some $\omega$-bromoaldehydes using ketone cyanohydrins as the cyanide source. This reaction allows us to achieve a double objective: prepare chiral cyanohydrins from ketones of $(S)$ configuration and the synthesis of $\omega$ bromocyanohydrins. These cyanohydrins are precursors of different oxygenated and nitrogenated heterocycles. It is worth noting that using this methodology $(S)$-pipecolic acid and other alkaloid derivatives have been prepared. In addition, starting from the corresponding cyanohydrins of different $\omega$ bromoaldehydes we have tackled the synthesis of heterocycles of more than six members, and have been able to isolate optically active azepane and azopane derivatives.

\section{References and Notes}

\section{Aminolysis and ammonolysis reactions}

1. García, M.J. Rebolledo, F., Gotor, V. "Lipase-catalyzed Aminolysis and Ammonolysis of $\beta$ Ketoesters. Synthesis of Optically Active $\beta$-Ketoamides”. Tetrahedron, 1994, 50, 6935-6940.

2. Puertas, S., Rebolledo, F., Gotor V. "Enzymatic Aminolysis and Ammonolysis of Dimethyl 3- 
Hydroxyglutarate. Synthesis of (R)-4-Amino-3-hydroxybutanoic acid". J. Org. Chem., 1996, 61, 6024-6027.

3. Alfonso, I., Astorga, C., Rebolledo, F., Gotor, V. "Sequential Biocatalytic Resolution of (土)-transCyclohexane-1,2-diamine. Chemoenzymatic Synthesis of an Optically active Polyamine”. J. Chem. Soc. Chem. Commun., 1996, 2471-2472.

4. Alfonso, I, Rebolledo, F., Gotor, V. "Chemoenzymatic Synthesis of two Optically Active Hexaazamacrocycles". Tetrahedron: Asymmetry, 1999, 10, 367-374.

5. Sánchez, V.M., Rebolledo, F., Gotor, V. “Candida antarctica Lipase Catalyzed Doubly Enantioselective Aminolysis Reactions. Chemoenzymatic Synthesis of 3-Hydroxypyrrolydines and (4Silyloxi)-2-oxopyrrolydines with two Stereogenic Centers”. J. Org. Chem., 1999, 64, 1464-1470.

\section{Chemoselective Transformations of Natural Products}

1. Moris, F., Gotor, V. "A Useful and Versatile Procedure for the Acylation of Nucleosides through an Enzymatic Reaction". J. Org. Chem. 1993, 58, 653-660.

2. Fernández, S., Ferrero, M., Gotor, V. Okamura W. "Selective Acylation of A Ring Precursors of Vitamin D Using Enzymes in Organic Solvents". J. Org. Chem., 1995, 60, 6057-6061.

3. Garcia-Alles, L.F., Magdalena, J., Gotor, V. 'Synthesis of Purine and Pyrimidine 3'-Amino-3'deoxy and 3'-Amino-2',3'-dideoxyxilonucleoxides”. J. Org. Chem., 1996, 61, 6980-6986.

4. Ferrero, M., Fernández, S., Gotor, V. "Selective Alkoxycarbonylation of A Ring Precursors of Vitamin D Using Enzymes in Organic Solvents. Chemoenzymatic Synthesis of 1 $\alpha, 25$ Dihydroxyvitamin D 3 C-5 Ring Carbamate Derivatives". J. Org. Chem., 1997, 62, 4358-4363.

5. Magdalena, J., Fernández, S., Ferrero, M., Gotor, V. 'Chemoenzymatic Synthesis of Novel 3' and 5' Carbazoyl Nucleoside Derivatives. Regioselective Preparation of 3' and 5'-Alkylidencarbazoyl Nucleosides". J. Org. Chem., 1998, 63, 8873-8879.

Reactions using (R)-Oxynitrilase

1. Menendez, E., Brieva, R., Rebolledo, F., Gotor, V. "Optically Active $(S)$-Ketone- and $(R)$ Aldehyde-cyanohydrins via an $(R)$-Oxynitrilase-catalysed Transcyanation. Chemoenzymatic Synthesis of 2-Cyanotetrahydrofuran and 2-Cyanotetrahydropyran". J. Chem. Soc. Chem. Commun., 1995, 989-990.

2. Nazabadioko, S., Pérez, R., Brieva, R., Gotor, V. "Chemoenzymatic Synthesis of (S)-2Cyanopyperidine, a Key Intermediate in the Route to $(S)$-Pipecolic Acid and 2-Substituted Piperidine Alkaloids". Tetrahedron: Asymmetry, 1998, 9, 1597-1604.

*Note: Translation by the Editorial Staff. 